\title{
Realities and Challenges of a Five Year Follow Up of Mother and Child Pairs on a PMTCT Program in Zimbabwe
}

\author{
E.N. Kurewa ${ }^{*}, 1$, G.Q. Kandawasvika ${ }^{2}$, F. Mhlanga ${ }^{3}$, M. Munjoma ${ }^{3}$, M.P. Mapingure ${ }^{4}$, \\ P. Chandiwana ${ }^{1}$, M.Z. Chirenje ${ }^{3}$, S. Rusakaniko ${ }^{4}$ and B. Stray-Pedersen ${ }^{5}$
}

\author{
${ }^{1}$ Letten Foundation Research Center, No.3 Everrett Close, Harare Zimbabwe. \\ ${ }^{2}$ Department of Paediatrics, College of Health Sciences University of Zimbabwe Medical School, Box A178, Harare, \\ Zimbabwe \\ ${ }^{3}$ Department of obstetrics and gynaecology, College of Health Sciences University of Zimbabwe Medical School, Box \\ A178, Harare, Zimbabwe \\ ${ }^{4}$ Department of Community Medicine, College of Health Sciences University of Zimbabwe Medical School, Box A178, \\ Harare, Zimbabwe \\ ${ }^{5}$ Department of Obs and Gynae, Division of women's health, Rikshospitalet, University of Oslo, Norway
}

\begin{abstract}
Background: Complete follow up is an essential component of observational cohorts irrespective of the type of disease.

Objectives: To describe five years follow up of mother and child pairs on a PMTCT program, highlighting loss to follow up (LTFU) and mortality (attrition).

Study Design: A cohort of pregnant women was enrolled from the national PMTCT program at 36 weeks gestational age attending three peri urban clinics around Harare offering maternal and child health services. Mother-infant pairs were followed up from birth and twice yearly for five years.

Results: A total of 479 HIV infected and 571 HIV negative pregnant women were enrolled, 445(92.9\%) and 495(86.6\%) were followed up whereas $14(3.0 \%)$ and $3(0.5 \%)$ died in the $1^{\text {st }}$ year respectively; RR $(95 \% \mathrm{CI}) 5.3(1.5-18.7)$. At five years 227(56.7\%) HIV infected and 239(41.0\%) HIV negative mothers turned up, whereas mortality rates were 34 and 7 per 100 person years respectively. Birth information was recorded for 401(83.7\%) HIV exposed and 441(77.2\%) unexposed infants, $247(51.6 \%)$ and $232(40.6)$ turned up in the first year whilst mortality was $58(12.9 \%)$ and $22(4.4 \%)$ respectively, RR (95\%CI) 3.2(2.0-5.4). At five years 210(57.5\%) HIV exposed and 239(44.3\%) unexposed infants were seen, whilst mortality rates were 53 per 1000 and 15 per 1000 person years respectively. Mortality rate for HIV infected children was 112 compared to 21 per 1000 person years for the exposed but uninfected.

Conclusion: HIV infected mothers and their children succumbed to mortality whereas the HIV negatives were LTFU. Mortality rates and LTFU are high within PMTCT program.
\end{abstract}

Keywords: HIV, Follow up, Mortality, PMTCT.

\section{INTRODUCTION}

In 2000, WHO responding to the HIV pandemic recommended that interventions to prevent mother to child transmission (PMTCT) of HIV interventions with a four pronged approach based on antenatal voluntary counselling and testing(VCT) of all pregnant women and that antiretroviral (ARV) treatment should be integrated within all maternal and child health $(\mathrm{MCH})$ programs. PMTCT interventions were accepted in Zimbabwe as a national program in 2001 , whilst it is estimated that up to $12 \%$ of infants in Zimbabwe are infected with HIV [1-5].

*Address correspondence to this author at the Letten Foundation Research Center, No.3 Everrett Close, Harare Zimbabwe; Tel: 0047 21383634;

E-mail: enkurewa@hotmail.com
The success of PMTCT is based on complete follow up of mothers and infants to ensure maximum compliance and retention that allows correct timing of interventions. The major challenge in the successful implementation of the PMTCT protocol is loss to follow up (LTFU) and mortality (attrition). Attrition results in a weak link between PMTCT initiatives of identifying and monitoring HIV exposed and unexposed infants in the under 5 child health clinics [6-12]. PMTCT outcomes reported are based mainly on those mother child pairs who retained within programs, leading to limited information of those that died and the ones that are LTFU.

Literature shows that high attrition within PMTCT programs could be more of LTFU than of mortality. Cumulative losses in sub-Saharan African PMTCT programs are estimated to range from $20-28 \%$ during antenatal care, up to $70 \%$ at four months postpartum and close to $81 \%$ at six 
months after birth [7,9,13-18]. Complete follow up is an essential component of all observational cohorts irrespective of the type of disease [19].

Our study, Better Health for The African Mother and Child (BHAMC) recruited pregnant women from the Zimbabwe national PMTCT program before CD4 monitoring and long term ARV therapy was readily available and affordable in the country. In this paper we describe 5 years follow up of mothers and children within a PMTCT intervention with a special focus on attrition. The aim is to highlight the reality of following mother and child pairs beyond the stipulated PMTCT 24 months. Identification of the time points when high attrition occurs will guide planning and targeting of interventions to maximize compliance which is needed to improve mother's health and child.

\section{MATERIALS AND METHODS}

Design of the study: A cohort of mother and child pairs was recruited from the national PMTCT program and followed up for five years.

Study sites: Three peri urban clinics around Harare the capital city of Zimbabwe, namely; Epworth; St Marys and Seke North. These are primary health care settings offering maternal and child health services.

Study population: Pregnant women booked at the respective Antenatal Clinics (ANC), who have gone through the national PMTCT (VCT) were followed up together with their index children from birth up to 5 years.

Inclusion criteria: Pregnant women from 36 weeks of gestation coming through the national PMTCT program. Pregnant women with known complications and who not had to deliver at a primary health care setting were excluded.

Sample size: The intention was to recruit 300 HIV positive and 600 HIV negative pregnant women allowing for $10 \%$ LFU. 'EPISTAT' program was used to calculate the sample size.

Baseline assessments of mothers: A questionnaire sourcing socio demographic, life styles and living conditions, obstetric and reproductive history, contraception and knowledge of STI/HIV signs and symptoms was administered to the women at enrolment. In addition to routine antenatal care women were screened for other sexually transmitted infections and reproductive tract infections (RTI)s. Those found infected were treated and encouraged to inform their sexual partners to come for treatment which was given for free.

A locator form was used to document the physical and postal address of the mother or care giver and next of kin details were obtained for those who consented to home visits, where available contact telephone numbers were obtained for follow up purposes. Trained counselors residing in the same community were responsible for the follow up of those participants who missed their scheduled visits. All transport costs were reimbursed, whilst all clinical care expenses related to any clinical event were supported by the project.

Intervention: At birth HIV infected mothers received a single 200-mg Nevirapine dose at the onset of labor, and their infants received a single 1-2 mg Nevirapine dose within 72 hours of delivery (sdNVP). Nevirapine was provided by the government of Zimbabwe under the national PMTCT program.

Support groups were introduced during the second year of follow up where women met clinician once every week. At each study clinic women were encouraged to form one group for both HIV infected and negative women. They chose a convenient day where they would meet at a specified time for up to 2 hours and a coordinator amongst themselves to act as a link person between them and the researchers.

Health education talks were given to the women interacting with the research nurses, counselors, psychologist and sociologist to respond to their concerns in a participatory manner. Women were also given an opportunity to suggest topics which they felt relevant to their day to day lives. They were encouraged to bring their spouses and significant members like mothers' in law or any caregiver for the index child where the mother had been lost.

Note: Enrolment was done during the time when prolonged ARVs were not readily available in the country except for the sdNVP, whereas CD4 counts were not routinely done at government institutions due to prohibitive costs. However over the follow up period when they became available and affordable the project facilitated referral for the HIV infected and exposed children to access both CD4 counts and ARVs when indicated from the government opportunistic clinics (OI).

Follow up: This was conducted within the same study clinics where maternal and child health services are provided. A separate room was provided for the study procedures throughout the follow up period. Mothers were followed up according to their expected date of term (EDT) to ascertain site and mode of delivery and for the HIV infected to ensure provision of NVP. A birth form was used to document birth information; onset of labour, time of rupture of membranes, duration of labor, collection of venous blood and for the HIV infected women, time of ingesting Nevirapine tablet.

All mothers and their infants were followed up for 6 weeks, four and nine months and every six months thereafter for five years. Mother and child health status was assessed and documented in the appropriate form according to the age after birth. Infant feeding practices were assessed and documented at each visit. HIV infected mothers and their exposed infants were given prophylactic cotrimoxazole. For the exposed infants cotrimoxazole would be stopped if they tested negative for HIV.

HIV infected mothers had a general physical and gynaecological examination performed together with CD4 counts that were assessed every six months. Those with a low CD4 count $(<$ less than $200 \mathrm{~mm} / 1$ would be send for a chest $\mathrm{x}$ - ray, blood chemistry; liver function tests (LFTs), Urea and electrolytes (U \& Es and full blood count (FBC) for easy access and referral to the government opportunistic infection (OI) clinics for ARV therapy.

Outcome information was when the study participants were considered to be alive; they turned up for their scheduled visit, they could be contacted over the phone, 
could be seen at home or if a spouse or close relative, or care giver of the child could confirm that they were alive during the period under observation. They were considered deceased if a spouse, close relative, landlord or neighbor seen at their house or nearby confirmed verbally or medical records that confirmed their death.

In the case where the visit was unsuccessful, they were not proven as deceased or alive and follow up visits would have been repeated for unspecified times and all channels of contact exhausted. They would then be considered as LFU.

Children's follow up: Follow up of the commenced from birth for both HIV exposed and unexposed neonates was carried out. Birth information was recorded in an infant birth form by the midwife regarding status of the neonate at birth; alive or still born, Apgar score, physical examination including anthropometrical measurements and collection of a cord blood sample for the HIV exposed infants.

Thereafter venous blood was collected at each follow up visit; 6 weeks, 4 and 9 months for HIV- DNA PCR test using cell pellets by prototype Roche Amplicor version 1.5 qualitative PCR assay (Roche Diagnostic Systems). At six weeks cotrimoxazole suspension was given to all HIV exposed infants until they were screened and found negative. At each visit a health status was documented in the appropriate form according to age at visit after a complete physical examination of the infant together with anthropometric measurements and feeding practices.

A Bailey Infant Neurological development test (BINS) was performed from 3 to 24 months. Thereafter the children continued to have a general health status assessment every six months performed by the study paediatrician. HIV exposed infants were screened for HIV one month after cessation of breastfeeding and from 15 months onwards.

HIV infected infants had a full blood count test (FBC), blood chemistry tests and CD4 counts every six months. Where the infants indicated were referred to OI clinics to be commenced on ARVs.

Infants were classified at birth according to maternal HIV status that was determined at approximately 36 weeks of gestation and this status was maintained for the analysis of this paper. At each scheduled visit information was sourced from the mother or care giver about the health status of the infant. Those who did not turn up for the scheduled visit were followed up to ascertain reason for defaulting. If found they would be rescheduled for the next visit.

An infant was considered as LTFU if he was not brought in for the stipulated visit and not declared as deceased by either the parents or caregiver. The infants were considered dead through evidence from verbal autopsy for the baby given by either the mother or care giver or when available clinical records were analyzed to ascertain cause of death.

Attrition, (defined as the total sum of those who were LTFU and those that died) was assessed at each scheduled visit over the five years.

Ethical Considerations: Both the Medical Research Council of Zimbabwe and the Ethical Review Committee in Norway approved the study. Mothers who agreed to participate gave an informed written consent that covered themselves and their index babies to be followed up from birth.

Statistical Analysis: Data were entered and analyzed using SPSS and STATA Version 9 from StataCorp Texas USA. All mothers enrolled in pregnancy were assessed whilst all live born infants were available for analysis. In the case of multiple births only the first born was included to avoid mixing up of the children. Baseline characteristics were compared using Pearson $\mathrm{X}^{2}$ test for categorical variables and independent sample student's $t$ test for continuous variables.

Follow up of mothers and infants reflecting on those seen, LFU and deceased at each scheduled visit from birth to 5 years is demonstrated by a flow diagram (Figs. 1, 2).

Scheduled visits were combined from birth up to one year to summarize those ever seen and those who were LTFU and the ones that died as outcome information. Risk ratios with their $95 \%$ CI of dying or being lost to follow up were estimated over the $1^{\text {st }}$ year and at five years using maternal HIV status at enrolment.

Follow up time was measured in months from enrolment up to 60 months ending at the earliest month of death and ending at the last follow up visit, date of last contact. The statistical significance threshold was $5 \%$, whilst all reported $\mathrm{p}$ values were 2 sided.

\section{RESULTS}

A total of 1050 pregnant women were enrolled from 36 weeks of gestation being 479 HIV infected and 571 HIV negatives. Their mean ages were $26.0 \mathrm{yrs}$ (s.d 4.9) for the HIV infected and $23.5 \mathrm{yrs}(\mathrm{s.d} 4.8)$ for the HIV negatives and reported mean pregnancy (s.d) $1.9(0.41)$ and $1.6(0.5)$ respectively $\mathrm{p}<0.001$. Birth information revealed that 7 infants were stillborn, 5 from the HIV infected and 2 from HIV negative mothers; whereas $4(0.8 \%)$ HIV infected women and 11(1.9\%) HIV negative women refused continued participation after birth $\mathrm{p}<0.001$.

Birth information was obtained from 851(81.0\%) mothers, 406 HIV positive and 445 HIV negatives that gave birth within the study sites whilst 11 mothers delivered at referral hospitals due to recorded complications. A total of $318(66.4 \%)$ mother infant pairs took NVP. Three hundred and ninety one (81.6\%) HIV exposed children were screened for HIV over the five years and 97 of them were infected with HIV through MTCT giving an overall transmission rate of $21.8 \% \mathrm{CI}(17.8-25.8)$. This is fully described in another paper.

Mothers' follow up: Over the first year more mothers were LFU among the HIV negatives 76 (13.4\%) compared to the HIV infected $34(7.3 \%) p=0.012$. In the same period more mothers were lost due to mortality among the HIV infected compared to the HIV negatives. At five years LFU was not different among the HIV infected and negative mothers, odds ratio (OR) $(95 \% \mathrm{CI}) 1.6(0.9-2.7)$. Mortality for mothers continued to rise over the five years regardless of their HIV status. Risk ratios for LFU and mortality for HIV infected and negative mothers are shown in Table $\mathbf{1}$. 


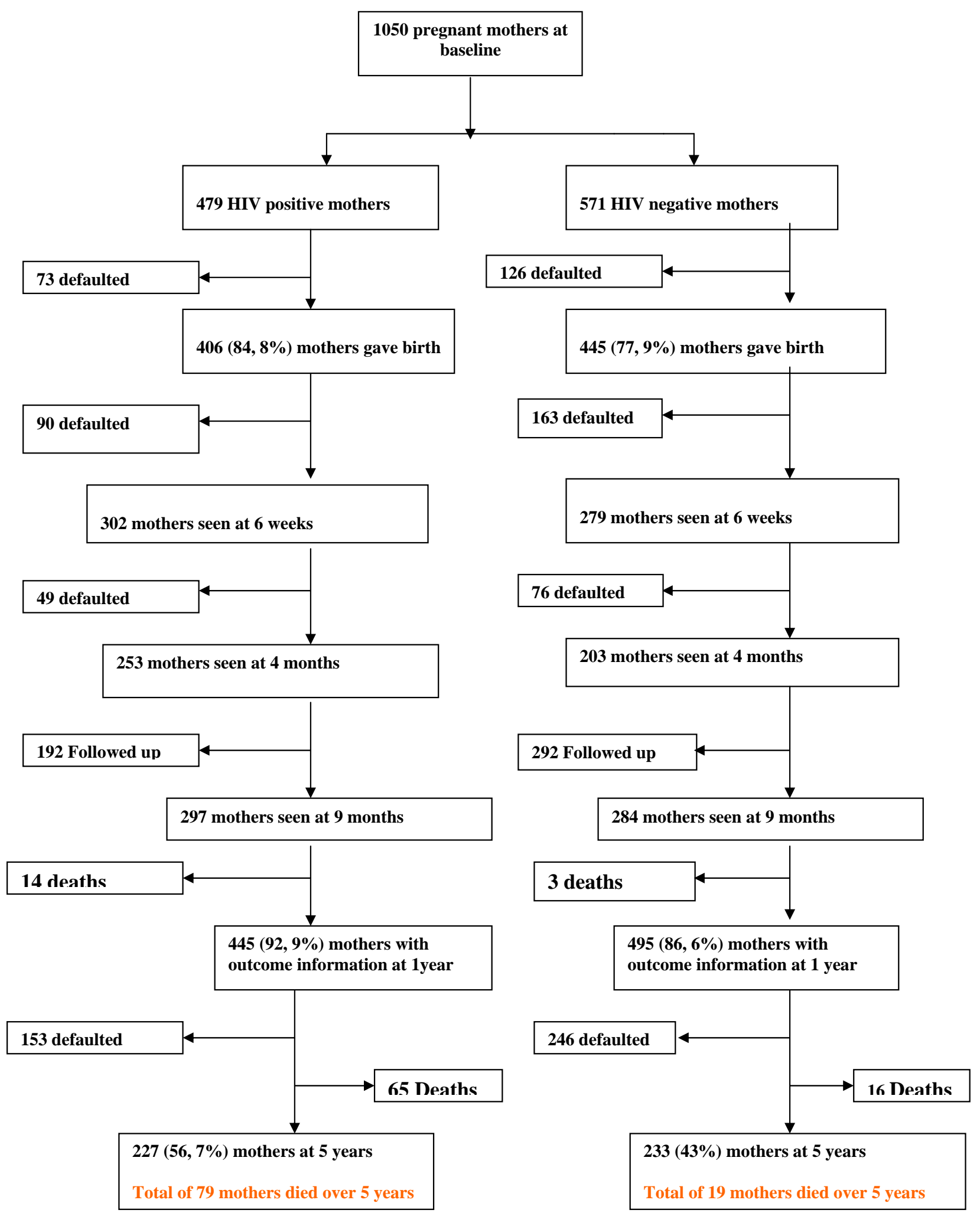

Fig. (1). Mothers follow Up over 5 years.

Figs. (1, 2) illustrate flow of mothers and children during the five years of follow up based on the maternal HIV status at enrolment. A total of 253(52.8\%) HIV positive mothers consistently showed up at each of the scheduled follow up visits compared to 203(35.5) HIV negatives $p<0.001$.
Throughout the follow up period, 369(77.0\%) HIV infected and 480(84.1\%) HIV negative mothers were seen both in the first year and over the five years respectively. A total of 187(46.8\%) HIV infected and 166(30.1) HIV negative mothers were coming consistently at each of the scheduled 11-12 visits over the five years $\mathrm{p}<0.001$. 


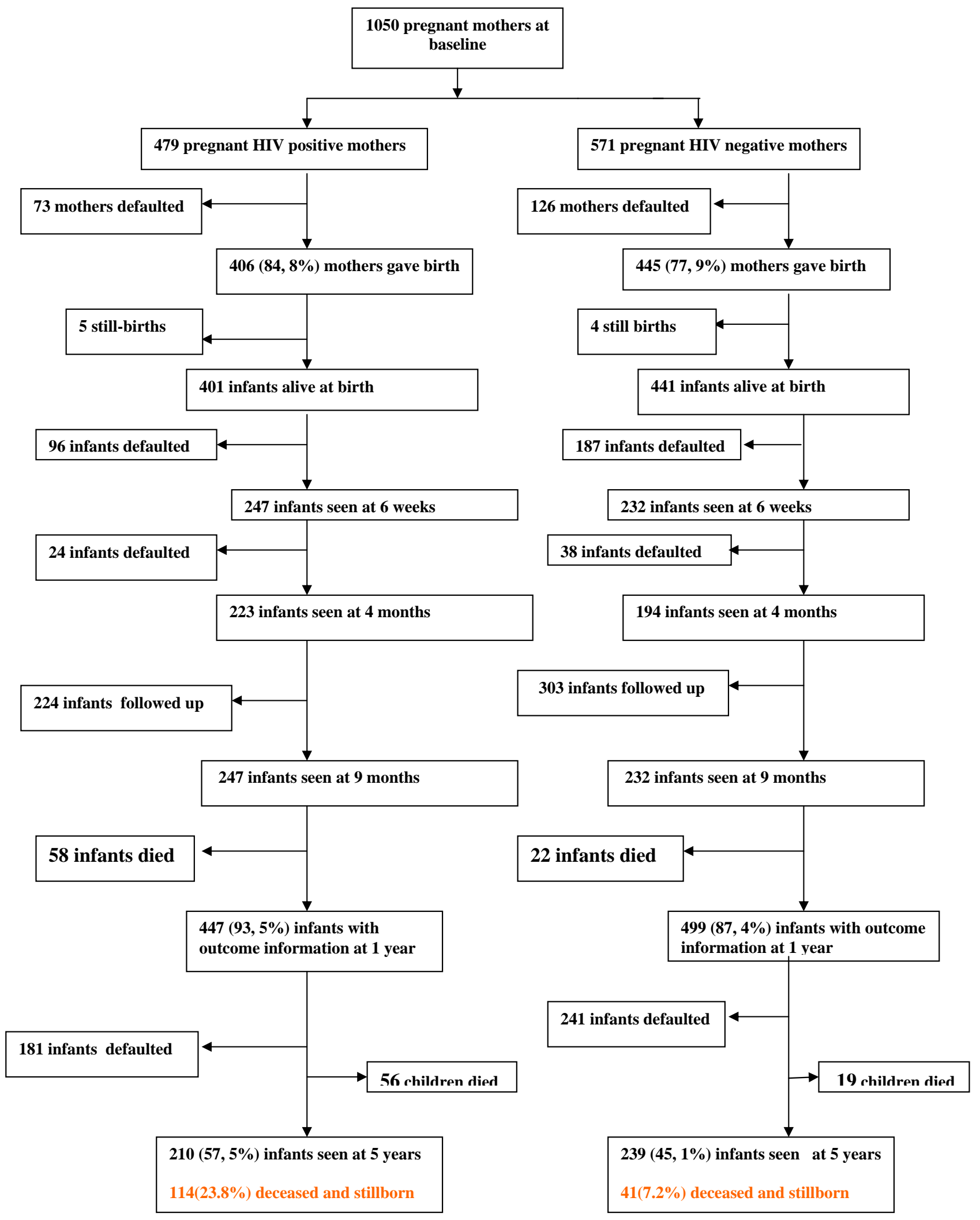

Fig. (2). Infants follow up over 5 years.

A total of $227(56.7 \%)$ HIV infected mothers responded to the 60 months questionnaire compared to 233(43.0) HIV negatives $p=0.013$. Over the five years, 305(63.8\%) HIV infected mother and child pairs turned up at different time points compared to $459(80.4 \%)$ HIV negatives $\mathrm{p}>0.05$ whilst
$36(7.5 \%)$ HIV infected mother and child pairs died compared to $4(0.7 \%)$ among the HIV negatives $\mathrm{p}<0.001$.

Follow up of children: A total of 247 (58.7\%) HIV exposed and 232 (43.5\%) HIV unexposed children showed 
Table 1. Risk of LFU and Mortality of HIV Positive and Negative Mothers and their Children

\begin{tabular}{|c|c|c|c|c|}
\hline & $\begin{array}{l}\text { LFU and } \\
\text { Mortality }\end{array}$ & $\begin{array}{c}\text { HIV }^{\text {POSITIVE }} \\
\mathbf{N}=\mathbf{4 7 9}\end{array}$ & $\begin{array}{c}\text { HIV }^{\text {NEGATIVE }} \\
\mathbf{N}=\mathbf{5 7 1}\end{array}$ & $\begin{array}{c}\text { Risk } \\
\text { Ratio }\end{array}$ \\
\hline \multicolumn{5}{|l|}{ Mothers } \\
\hline \multirow[t]{2}{*}{ Visit during first year } & LFU & $34(7.3 \%)$ & $76(13.4 \%)$ & $1.96(1.28-2.99)$ \\
\hline & Deceased & $14(3.1 \%)$ & $3(0.6 \%)$ & $5.32(1.52-18.66)$ \\
\hline \multirow[t]{2}{*}{ Visit over five years } & LFU & $25(5.2 \%)$ & $54(9.5 \%)$ & $1.63(0.99-2.66)$ \\
\hline & Deceased & $79(16.5)$ & $19(3.3)$ & $5.52(3.29-9.27)$ \\
\hline \multicolumn{5}{|l|}{ Children } \\
\hline \multirow[t]{2}{*}{ Visit during first year } & LFU & $31(7.4 \%)$ & $72(13.1 \%)$ & $1.89(1.22-2.95)$ \\
\hline & Deceased & $58(12.9 \%)$ & $22(4.4 \%)$ & $3.22(1.94-5.36)$ \\
\hline \multirow[t]{2}{*}{ Visit over five years } & LFU & $16(3.3 \%)$ & $51(8.9 \%)$ & $2.32(1.30-4.14)$ \\
\hline & Deceased & $114(23.8 \%)$ & $41(7.2 \%)$ & $3.82(2.60-5.59)$ \\
\hline
\end{tabular}

up for the one year visit $\mathrm{p}<0.001$ whilst $58(12.1 \%)$ compared to $22(3.9 \%)$ died respectively $\mathrm{p}<0.001$.

There were 223(52.9\%) HIV exposed compared to 194(35.3\%) unexposed children turning up consistently for each of the scheduled (4) visits over the first year of follow up $\mathrm{p}=0.002$ (Fig. 2).

Throughout the five years of follow up, 391(82.5\%) HIV exposed children were screened for HIV at various time points and $97(24.8 \%)$ of them were HIV seropositive. Among the HIV infected infants, 23(23.7\%) died in the first year, whilst 43/74(58.1\%) died after their first birthday.

Overall 5 years mortality of the HIV infected children was $66(68.0 \%)$, with mortality rates of 112 compared to 21 per 1000 person years among the HIV exposed but not infected children.

A significant difference was observed in the compliance of children by maternal HIV status at 5 years, with $210(57.5 \%)$ HIV exposed children turning up for scheduled visits compared to $239(45.1 \%)$ those born of HIV negative mothers, $\mathrm{p}<0.001$. A total of $137(37.5 \%)$ HIV exposed infants and $112(21.5 \%)$ HIV negative mothers consistently turned up for each visit throughout the five years, $\mathrm{p}<0.001$.

Cumulative 5 years child mortality was 114(23.8\%) among HIV exposed children and 41(7.2\%) for those not exposed, $\mathrm{p}<0.001$; mortality rates were 53 per 1000 and 15 per 1000 person years for the HIV exposed and unexposed respectively. During the five years of follow up $16(4.4 \%)$ HIV exposed and 51(9.6\%) unexposed children were not able to be traced at all $\mathrm{p}=0.001$.

\section{DISCUSSION}

This study highlights a natural flow of mother and child pairs followed up from a PMTCT program before routine CD4 counts were affordable and there was availability of long term ARV therapy in low resource settings. Less than $10 \%$ of both mothers and children were completely LTF regardless of HIV status. Outcome information was obtained for up to ninety percent of both HIV infected and negative mothers and their children whilst actual retention was fifty and forty percent respectively over the five years.
Mothers who did not have birth information were mostly referral cases who developed complications during birth and were referred or transferred for higher level of care at the central hospitals for further management whereas some had decided not to give birth at the study clinics. Others were those who later decided not to give birth elsewhere outside the study sites.

Our retention rate is higher compared with other African studies although few of them have continued PMTCT follow up for more than 2 years [6]. NVP intake for our cohort was higher than the national and other regional reports at that time. This could be due to the extra health education and close monitoring of participants within research initiatives [20] and the 2006 ZDHS.

Attrition was mainly due to high mortality among the HIV infected mothers and their children, whilst the HIV negative mothers and their children were LTFU. Mortality of the HIV infected mothers and exposed children was higher in the first year but with time the HIV negative mothers and children were also dying [21].

Child mortality decreased with age whereas that of the mothers increased with time. This explains that the high number of orphaned children in Zimbabwe as HIV exposed children survive more than their HIV infected parents as reflected in the Zimbabwe demographic health survey.

Other factors besides HIV infection could be influencing this gradual increase in mortality observed even among the HIV negatives too. Mortality rates are consistent with what other studies in the region have reported from the stipulated/recommended PMTCT follow up of two years after birth [1,6,13,15,22-25].

Mortality of the HIV infected children was lower compared to other reports where it ranged from 50 to $80 \%$ over two years $[6,15,23,26,27]$. This could be due to the early provision and constant supply of prophylactic cotrimoxazole by the study and availability of a paediatrician who offered timeous management and referrals for ARV therapy. These mortality figures are quite representative as we managed to get over ninety percent outcome information compared to reports from other studies [26, 28,29]. 
Mortality of the HIV infected children reflects the need for early provision of ARVs. This is in line with observations and concerns that HIV exposed children are turning up late for HIV screening, when they are already sick being in the AIDS stage $[12,14,30]$. This is attributed to the weak link and integration of PMTCT interventions with the traditional under five child health clinics. This would provide an easy access and early identification of HIV exposed children and those found infected would be referred to OI clinics for further management.

The omitted visit at birth meant a missed opportunity for NVP intake for the HIV infected mother and exposed infant and this compromized the outcome of infections averted and generalizability to other studies. Estimation of MTCT rates of HIV from birth throughout the breastfeeding period is distorted when exposed children are screened for HIV at different time points. Other studies have reported that inadequate follow up of infants post delivery leads to poor quantification of infections averted [31].

Six weeks is an existing cardinal established WHO stipulated visit for women's post natal examination and that of their infants. Women who missed the opportunity to have a pap smear taken which is important for cervical screening of cancer regardless of one's HIV status. The HIV negative mothers not showing up for scheduled visits missed the HIV screening test thereby affecting the quantification of HIV incidence rate for this cohort. With only thirty percent being consistently screened for HIV it becomes difficult to generalize the results as they were not the same women who were being screened at the same time points.

LTFU of children during this period when they are supposed to be attending the under 5 child health clinics for growth monitoring and immunizations has been reflected in the low vaccination coverage reported in the country over the same period [20].

More than twenty percent of the outcome information obtained for the HIV infected and exposed children was on mortality whilst for the HIV negatives it was LTFU. HIV infected mothers need to have CD4 counts every six months whilst the HIV negatives should appreciate the importance of taking an HIV test at least every six months or yearly.

Mothers should be given health education on the importance of growth monitoring and immunization of children emphasizing complete vaccination regardless of maternal HIV status. Mortality figures for mothers and children should be included in the HIV prevention messages in the respective communities. This will assist couples to make informed decisions regarding HIV infection and future pregnancies.

The strength of this study is the valuable information of mothers and children documented over five years which is useful for planning of maternal and child health interventions. The $30 \%$ HIV infected children surviving after their $5^{\text {th }}$ birthday require a targeted health care plan according to their specific needs besides provision of HAART. We conclude that HIV infected mothers and exposed children mainly die due to mortality, whilst the HIV negatives and their children are LTFU. Attrition over five years was similar regardless of maternal HIV status, meaning that factors other than HIV status could be influencing it. Outcome measures are compromized because of the irregular compliance as different mothers and children turn up at different time points. Mortality and HIV incidence figures should be disseminated to the concerned respective communities for them to make informed decisions regarding compliance.

Emphasis on complete follow up care regardless of HIV status should be part of all health care programs. We support integration of PMTCT initiatives into HIV health care services for a holistic approach to service provision. There is need to ascertain factors that influence high attrition within follow up studies.

This information will aid in informing program coordinators and health care policy makers to improve tracking methods and directing more effort towards targeting those time points where attrition is indicated to be highest.

The main limitation is that we did not describe the predictors of this attrition and that we lacked a comparison group that followed up mother and child pairs from a PMTCT program over 5 years.

\section{CONFLICT OF INTEREST}

The authors declare no conflict of interest

\section{ACKNOWLEDGEMENTS}

The authors gratefully acknowledge the women and children who participated in this study. Special thanks to the local clinic community leaders, authorities, study nurses, counselors, laboratory and support staff at the College of Health Sciences, Department of Obstetrics and Gynaecology and Paediatrics. A special mention goes to the Letten Foundation of Norway and Professor Letten F Saugstad herself for funding this study.

\section{REFERENCES}

[1] Iliff PJ, Piwoz EG, Tavengwa NV, et al. Early exclusive breastfeeding reduces the risk of postnatal HIV-1 transmission and increases HIV-free survival. AIDS 2005; 19(7): 699-708.

[2] Mahomva A, Greby S, Dube S, et al. HIV prevalence and trends from data in Zimbabwe, 1997-2004. Sex Transm Infect 2006; (Suppl 1): i42-7.

[3] Nathoo K, Rusakaniko S, Zijenah LS, et al. Survival pattern among infants born to human immunodeficiency virus type-1 infected mothers and uninfected mothers in Harare, Zimbabwe. Cent Afr J Med 2004; 50(5-6): 57.

[4] Newell ML, Brahmbhatt H, Ghys PD. Child mortality and HIV infection in Africa: a review. AIDS 2004; 18(Suppl 2): s27-34.

[5] Perez F, Orne-Gliemann J, Mukotekwa T, et al. Prevention of mother to child transmission of HIV: evaluation of a pilot programme in a district hospital in rural Zimbabwe. BMJ 2004; 329(7475): 1147-50.

[6] Arrive E, Kyabayinze DJ, Marquis B, et al. Cohort profile: the paediatric antiretroviral treatment programmes in lower-income countries (KIDS-ART-LINC) collaboration. Int J Epidemiol 2008; 37(3): 474-80.

[7] Moth IA, Ayayo AB, Kaseje DO. Assessment of utilisation of PMTCT services at Nyanza Provincial Hospital, Kenya. SAHARA J 2005; 2(2): 244-50.

[8] Msellati P, Hingst G, Kaba F, Viho I, Welffens-Ekra C, Dabis F. Operational issues in preventing mother-to-child transmission of HIV-1 in Abidjan, Cote d'Ivoire, 1998-99. Bull World Health Organ 2001; 79(7): 641-7.

[9] Painter TM, Diaby KL, Matia DM, et al. Sociodemographic factors associated with participation by HIV-1-positive pregnant women in an intervention to prevent mother-to-child transmission of HIV in Cote d'Ivoire. Int J STD AIDS 2005; 16(3): 237-42. 
[10] Paintsil E, Andiman WA. Update on successes and challenges regarding mother-to-child transmission of HIV. Curr Opin Pediatr 2009; 21(1): 94-101.

[11] De Baets AJ, Sifovo S, Pazvakavambwa IE. Early identification and care of HIV-exposed and HIV-infected children in rural Africa: the role of primary health care centers. J Acquir Immune Defic Syndr 2008; 48(2): 230-2.

[12] Meyers T, Moultrie H, Naidoo K, Cotton M, Eley B, Sherman G. Challenges to pediatric HIV care and treatment in South Africa. J Infect Dis 2007; 196: (Suppl 3): S474-81.

[13] Chilongozi D, Wang L, Brown L, et al. Morbidity and mortality among a cohort of human immunodeficiency virus type 1-infected and uninfected pregnant women and their infants from Malawi, Zambia, and Tanzania. Pediatr Infect Dis J 2008; 27(9): 808-14.

[14] Jones SA, Sherman GG, Varga CA. Exploring socio-economic conditions and poor follow-up rates of HIV-exposed infants in Johannesburg, South Africa. AIDS Care 2005; 17(4): 466-70.

[15] Sherman GG, Jones SA, Coovadia AH, Urban MF, Bolton KD. PMTCT from research to reality--results from a routine service [see comment]. S Afr Med J 2004; 94(4): 289-92.

[16] Stringer EM, Sinkala M, Stringer JS, et al. Prevention of motherto-child transmission of HIV in Africa: successes and challenges in scaling-up a nevirapine-based program in Lusaka, Zambia. AIDS 2003; 17(9): 1377-82.

[17] Stringer JS, Sinkala M, Goldenberg R, Vermund S, Acosta E. Monitoring nevirapine-based programmes for prevention of mother-to-child transmission of HIV-1. Lancet 2003; 362(9384): 667.

[18] Stringer JS, Sinkala M, Maclean CC, et al. Effectiveness of a citywide program to prevent mother-to-child HIV transmission in Lusaka, Zambia. AIDS 2005; 19(12): 1309-15.

[19] Mocroft A, Kirk O, Aldins P, et al. Loss to follow-up in an international, multicentre observational study. HIV Med 2008; 9(5): 261-9.

[20] ZDHS 2005/2006. Central Statistical Office Harare Z, 2007, editors. Zimbabwe Demographic and Health Survey 2005-2006.

[21] Kurewa EN, Gumbo FZ, Munjoma MW, et al. Effect of maternal HIV status on infant mortality: evidence from a 9-month follow-up of mothers and their infants in Zimbabwe. J Perinatol 2010; 30(02): 88-92.

[22] Abdool KS, Abdool KQ, Adhikari M, et al. Vertical HIV transmission in South Africa: translating research into policy and practice. Lancet 2002; 359(9311): 992-3.

[23] Coetzee D, Hilderbrand K, Boulle A, Draper B, Abdullah F, Goemaere E. Effectiveness of the first district-wide programme for the prevention of mother-to-child transmission of HIV in South Africa. Bull World Health Organ 2005; 83(7): 489-94.

[24] Jones SA, Sherman GG, Varga CA. Exploring socio-economic conditions and poor follow-up rates of HIV-exposed infants in Johannesburg, South Africa. AIDS Care 2005; 17(4): 466-70.

[25] Shetty AK. Perinatally acquired HIV-1 infection: prevention and evaluation of HIV-exposed infants. Semin Pediatr Infect Dis 2005 16(4): 282-95.

[26] Little K, Newell ML, Luo C, Ngongo N, Borja MC, McDermott P. Estimating the number of vertically HIV-infected children eligible for antiretroviral treatment in resource-limited settings. Int $\mathrm{J}$ Epidemiol 2007; 36(3): 679-87.

[27] Newell ML, Coovadia H, Cortina-Borja M, et al. Mortality of infected and uninfected infants born to HIV-infected mothers in Africa: a pooled analysis. Lancet 2004; 364(9441): 1236-43.

[28] Taha TE, Graham SM, Kumwenda NI, et al. Morbidity among human immunodeficiency virus-1-infected and -uninfected African children. Pediatrics 2000; 106(6): E77.

[29] van Kooten Niekerk NK, Knies MM, Howard J, et al. The first 5 years of the family clinic for HIV at Tygerberg Hospital: family demographics, survival of children and early impact of antiretroviral therapy. J Trop Pediatr 2006; 52(1): 3-11.

[30] De Baets AJ, Ramet J, Msellati P, Lepage P. The unique features of pediatric HIV-1 in sub-Saharan Africa. Curr HIV Res 2008; 6(4): 351-62.

[31] Rollins N, Little K, Mzolo S, Horwood C, Newell ML. Surveillance of mother-to-child transmission prevention programmes at immunization clinics: the case for universal screening. AIDS 2007; 21(10): 1341-7.

(C) Kurewa et al.; Licensee Bentham Open.

This is an open access article licensed under the terms of the Creative Commons Attribution Non-Commercial License (http://creativecommons.org/licenses/by-nc/ $3.0 /$ ) which permits unrestricted, non-commercial use, distribution and reproduction in any medium, provided the work is properly cited. 